\title{
De l'Aliya à l'immigration, ou la lecture d'un continuum migratoire
}

\author{
William BERTHOMIERE*
}

Ychouv', Aliya ${ }^{2}$, «Etat des Juifs", Israël, ces noms évoquent immanquablement l'histoire juive contemporaine à travers sa concrétisation, en un foyer national, un Etat. Au cours de ce siècle, à la fois fragment de l'histoire juive millénaire et source des tensions qui ont parcouru - et parcourent encore - le Moyen Orient, c'est une dynamique migratoire considérable que nous découvrons.

Notre propos vise à lire cette période sous l'angle d'un continuum migratoire ${ }^{3}$, témoin des dissonances et consonances dans le déroulement des mobilités dans «l'espace-temps des juifs ». ${ }^{4}$ Une lecture que nous réaliserons en prenant soin de caractériser les différents temps migratoires qui composent ce continuum : le « rassemblement des exilés » en cet «Etat des Juifs », concrétisation de la pensée du

\footnotetext{
* Doctorant à I'UMR MIGRINTER-IERS-TIDE, CNRS, Université de Poitiers, 95 avenue du Recteur Pineau, 86022 Poitiers Cedex, France.

1 « Nom donné à la communauté juive de Palestine et à la société qu'elle y construit avant la création de l'Etat ». Pour une information concise, cf. l'entrée "Yshouv » in A. Gresh et D. Vidal, Les 100 portes du Proche-orient, Autrement, Paris, 1992, pp. 296-297.

2 Mot hébreu signifiant littéralement la "montée » vers la terre d'Israël et qui est employé dans le langage commun pour signifier l'immigration juive en Israël (pluriel : aliyoth).

3 Le terme de continuum est, ici, pensé comme une visibilisation de la "communauté de destin» (le 'Hemshe'h Goyrl yiddish) telle que l'a défini H. Minczeles (dans son Histoire générale du Bund, p. 132) à savoir l'intervention des notions de continuité, de permanence, voire de pérennité. Nous concevons donc à travers l'idée de continuum, qui "recouvre le concept d'un espace ininterrompu», une sorte "d'harmonie migratoire » au sein de laquelle les dissonances et consonnances se font l'écho des rapports et interprétations des sociétés d'accueil, et des communautés qu'elles regroupent, forgeant ainsi l'expression de la mobilité juive.

4 Cf. Médam Alain , «Dans l'espace-temps des Juifs », Espaces et Sociétés, n 72, 1993, pp. 930.
} 
père fondateur du sionisme politique Théodore Herzl ${ }^{5}$, l'affaiblissement des dynamiques migratoires et la concomitante structuration de la «mosaïque Israël » et enfin les actuelles vagues en provenance d'ex-URSS et d'Ethiopie.

La lecture d'un tel continuum nous permettra d'éclairer les incidences de ces périodes dans l'édification de la société israélienne — d'une identité nationale — et par là-même, de comprendre comment l'acception de la diaspora est passée au cours de cette période de celle d'une métonymie de l'Etat d'Israël vers celle d'une entité à part entière. Révéler ce continuum migratoire constituera un élément de compréhension supplémentaire dans l'évolution des relations israélo-palestiniennes. Ce cheminement s'achèvera par un nécessaire regard sur la dialectique "centre-périphérie " - Israël/diaspora - à travers le prisme d'observation que constitue la territorialité afin d'avancer dans la compréhension des rôles et devoirs de ces deux entités.

\section{LA REUNION DES COMMUNAUTES AU SEIN D'UN FOYER NATIONAL}

Le 14 mai 1948, David Ben Gourion, proclame l'Etat d'Israël et réalise ainsi la vision qu'avait eue Herzl lors du Premier congrès sioniste. ${ }^{6}$ Le « retour » est enfin possible et il s'avère en fait surtout nécessaire, car d'une part, la détresse est forte au sein du peuple juif - et de la population mondiale en général - au lendemain de cette terrible guerre, et d'autre part, la menace est grandissante pour les communautés sises en Terre d'Islam, à la suite de la proclamation de l'Etat d'Israël. Soulignons que le conflit israélo-arabe trouve certes sa source dans la création de l'Etat puisque cette date est celle des premiers exodes de réfugiés palestiniens, mais ce conflit puise ses racines plus profondément dans les tensions entre Juifs et Arabes qui se sont intensifiées avec les premières aliyoth que la Palestine a connu entre 1882-1948, notamment après la déclaration Balfour. ${ }^{7}$ Nous pourrions résumer cette situation, qui a conduit aux tensions

5 Cf. Herzl Théodore, L'Etat des Juifs, La Découverte, Paris (Textes à l'appui), 1990. En 1897, Théodore Herzl a organisé le premier congrès sionsite à Bâle. Lors de ce rassemblement, les bases du projet sioniste ont été définies et c'est au cours de cette assemblée qu'a été crée I'Organisation Sioniste Mondiale. Pour une étude complète du sionisme et de la naissance du foyer national juif, voir notamment l'ouvrage d'Alain Dieckhoff, L'invention d'une nation : Israël et la modernité politique, Gallimard, Paris (nrf essais), 1993.

6 Herzl avait décrit son sentiment au lendemain du premier congrès à Bâle par les mots suivants : "Si je veux résumer le congrès de Bâle d'un mot - que je me garderai de prononcer en public — je dirai ceci : à Bâle j'ai fondé l'Etat juif. Si je disais cela aujourd'hui à haute voix, je déclencherais un rire universel. D'ici cinq ans peut-être, cinquante ans sûrement, chacun le comprendra. » Herzl Theodor, Journal 1895-1904. Le fondateur du sionisme parle, Calmann-Lévy, Paris (Diaspora), 1990, p 155

7 Le Ychouv a connu six grandes aliyoth avant 1948 : la première de 1882 à 1903, entre 20000 et 30000 migrants (dont les Biluhim, jeunes russes considérés comme les premiers migrants sionistes) ; la seconde de 1904 à 1914, entre 35000 et 40000 migrants (au cours de laquelle ont émigré les grandes figures d'Israël comme David Ben Gourion); la troisième de 1919 à 1924, 35000 migrants ; la quatrième de 1924 à 1931, 82000 migrants ; la cinquième de 1932 à 1938, 217000 migrants ; la sixième de 1939 à 1948, 153000 migrants. Ces données ne font état que des entrées et n'indiquent donc pas les départs qui ont été nombreux notamment au cours de la seconde aliya. 
actuelles, par les propos d'Alain Médam soulignant que « d'entrée de jeu les sionistes voulaient construire la Palestine en tant que foyer national tandis qu'il leur était accordé uniquement de créer un foyer national en Palestine. ${ }^{8}$

\section{Le rassemblement des exilés}

Le jeune Etat d'Israël voit donc au cours de ses premières années s'opérer le « rassemblement des exilés " (Kibboutz Galouyoth). Avec le soutien de l'Agence Juive', les immigrants viennent par milliers d'horizons divers caractérisés par l'exil, la misère et la mort.

\section{Un foyer pour les communautés rescapées d'Europe}

La création de l'Etat a immédiatement fait écho dans les communautés. Avec les 17000 entrées de juillet 1948, l'Etat voit son taux d'immigration mensuelle tripler par rapport à celui de mai. Cette dynamique va se poursuivre jusqu'en 1951 avec un flux qui oscille entre 10000 et 30000 arrivées par mois. Les rescapés des camps de concentration nazis, les réfugiés juifs d'Europe constituent la part la plus importante du flux migratoire. La dynamique migratoire est d'une telle intensité que fin décembre 1951, la population juive de l'Etat a déjà doublé. Aux 650000 personnes déjà installées lors de la proclamation de l'Etat sont venues s'ajouter plus de 685000 autres. Au sein de ce flux, de nombreuses communautés orientales étaient également présentes. Pour ces dernières, il s'est agi de véritables transferts de communautés : en 1950, l'Opération «Tapis Magique » voit le transfert de la quasi totalité de la communauté yéménite (50 000 personnes), entre 1948 et 1951 les Opérations «Ezra et Néhémie » permettent l'arrivée de la communauté d'Irak (130000 personnes).

A partir de 1952, comme le souligne la figure 1, l'intensité du flux s'atténue considérablement. Au cours de 1952-1953, environ 35000 entrées sont enregistrées contre 170000 en 1951. Cet affaiblissement est d'autant plus ressenti qu'Israël connaît en parallèle un mouvement d'émigration. Le solde migratoire est de 21000 personnes sur la période 1952-1954 (54000 arrivées pour 32000 départs). La récession migratoire s'explique essentiellement par la fin de l'évacuation des camps de "personnes déplacées", par l'interdiction d'émigrer que les démocraties populaires d'Europe imposent aux Juifs et par les difficultés rencontrées pour absorber cette vague massive d'immigration. Pour faire face à ces problèmes, le gouvernement israélien et

8 Medam Alain, Mondes Juifs. L'envers et l'endroit, PUF, Paris (La politique éclatée), 1991, p. 87.

9 L'agence Juive pour Israël a été créé en 1929 comme l'institution représentante des interêts juifs en Palestine au cours de la période mandataire. Cette institution a opéré au cours de cette période comme l'organe éxécutif de l'Organisation sioniste mondiale - créée par Th. Herzl lors du Premier congrès sioniste en 1897 - chargé du recrutement des immigrants et de leur intégration. Son action a été redéfinie après la création de l'Etat par la Loi de statut votée en 1952 par la Knesset.

REMI 1996 (12) 3 pp. 33-60 
Figure 1 : L'immigration en Israël suivant les continents d'origine entre 1948 et 1989

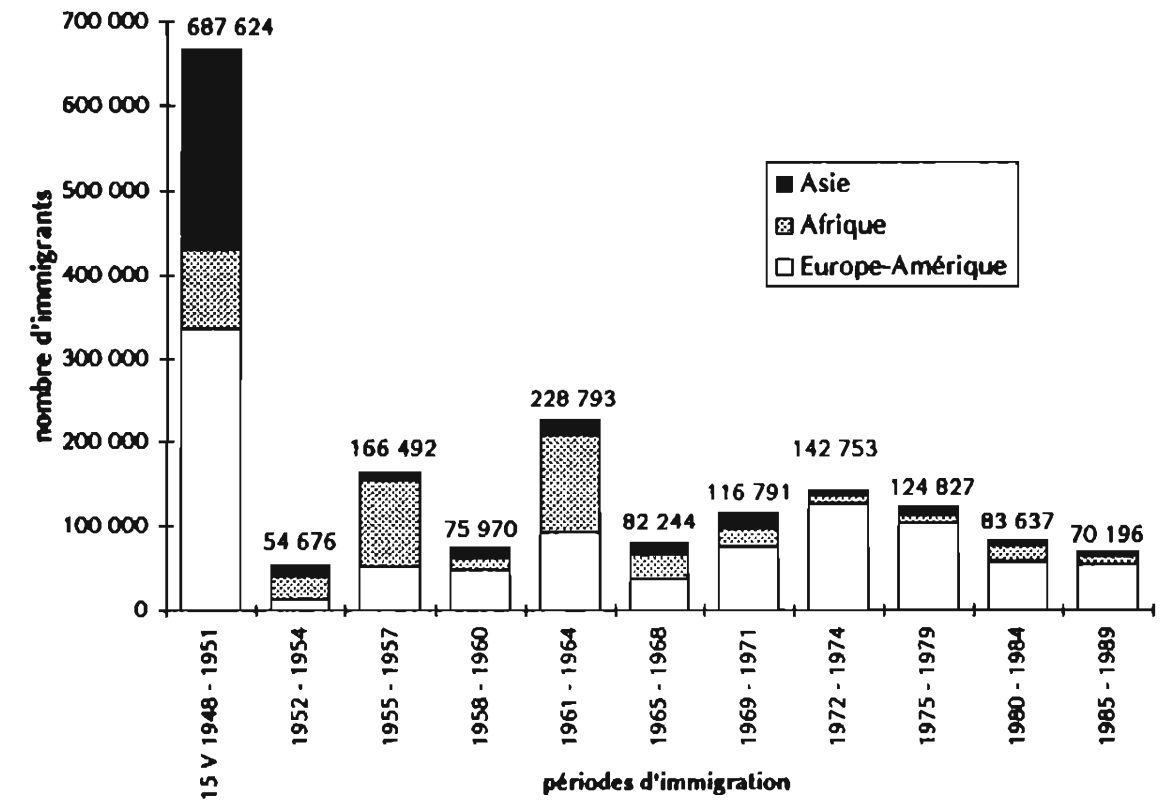

Source : Immigration to Israel 1993, Special series $N^{\circ} 973$, CBS, Jesuralem, p. 30.

(Les flux d'Océanie sont inclus dans ceux d'Europe-Amérique).

l'Agence juive avaient d'ailleurs mis en place une politique de sélection des immigrants qu'elle abandonna définitivement en 1955. Problèmes d'autant plus aigus qu'Israël devait assumer en parallèle le poids de fortes dépenses militaires.

\section{Un refuge pour les communautés sises en Terre d'Islam}

Pour ces communautés c'est dans les mouvements nationalistes en Afrique du Nord que nous trouvons le facteur déterminant de l'émigration vers Israël. Certes, l'attrait idéologique d'Israël a suscité un désir d'émigration puisqu'entre le 15 mai 1948 et le 31 décembre 1949, 47000 juifs ont réalisé leur aliya ${ }^{10}$ mais le plus fort de l'émigration s'est situé au cours des années cinquante et soixante. L'incertitude que représente l'après-colonisation et l'angoisse des persécutions qu'elle engendre poussent les communautés à émigrer. Quelques 27000 juifs de Tunisie et $197000 \mathrm{du}$ Maroc prennent la route d'Israël entre 1952 et $1964 .^{11}$ Cette dernière communauté

10 Chouraqui André, Histoire des Juifs en Afrique du Nord, Hachette, 1987, p. 482.

11 Notons que les juifs d'Algérie -citoyens français par le décret Crémieux de 1870- ont opté à $90 \%$ après l'indépendance de l'Algérie pour une émigration vers la France. Des Juifs de Tunisie se sont également tournés vers la France. Ainsi s'est opéré un réel partage de la communauté entre la France et Israël. (Pour des analyses sociologiques et géographiques des mobilités juives tunisiennes, voir les travaux de Bensimon-Donath (D.), L'intégration des Juifs nord-africains en France, Mouton, Paris, 1971 et de Simon (G.), L'espace des travailleurs tunisiens en France, Université de Poitiers, 1979, pp. 59-64). 
rencontre toutefois de plus en plus d'obstacles à l'émigration en raison de l'indépendance du Maroc (1956). Au cours de cette même année, Israël accueille environ 12000 juifs d'Egypte au lendemain de la campagne de Suez. ${ }^{12}$ A la même période, un regain d'immigration en provenance de Hongrie et surtout de Pologne est notable suite au relâchement des restrictions à l'émigration (environ 40000 personnes). Néanmoins Israël va progressivement vers une stabilisation de son flux d'immigration. Une seule exception viendra troubler l'accalmie qui caractérise la dynamique israélienne entre la crise de Suez et la Guerre des Six-jours : la poursuite d'une importante immigration marocaine qui voit l'arrivée de 130000 personnes entre 1961 et 1971.

Les fortes poussées migratoires à destination d'Israël seront par la suite principalement issues de la communauté soviétique - les juifs du silence - et dans une moindre mesure de Roumanie. ${ }^{13}$ La décennie soixante-dix en fut une parfaite illustration : sur les 267000 immigrants plus de la moitié étaient des Refuzniks - dont le plus célèbre d'entre eux était Nathan Charansky ${ }^{14}$ — qui avaient pu quitter l'Union sous la pression de la diaspora rassemblée sous le slogan : Let my People go.

\section{L'accueil dans l'urgence}

Les premiers immigrants, arrivés entre 1948 et début 1949, ont trouvé à se loger en partie dans les logements abandonnés par la population arabe à Jaffa, Lod, Haîfa, Akko et Jérusalem notamment. ${ }^{15}$ La plupart des nouveaux arrivants - de 1949 et 1951 - ont pu être accueillis dans les constructions réalisées pendant cette période (78 000 logements ${ }^{16}$ ), mais devant l'importance du flux, l'Etat a dû ériger des camps de tentes pour faire face. Le système du camp de transit plus connu sous le nom de Ma'abaroth, voit le jour en 1950. Plus de 120 Ma'abaroth sont installés à la périphérie des villes existantes ou dans les régions planifiées pour le développement.

12 Sur la période 1952-1960, 17500 juifs Egyptiens ont émigré en Israël. Notons que cette communauté était constituée de divers groupes juifs : une population de langue arabe enracinée depuis des siècles en Egypte, une population européenne d'origine séfarade dont certains avaient conservé leur langue, le Ladino, un groupe d'ashkénazes constitué de juifs ayant fui les pogroms de Russie et un groupe de Karaïtes (cf. Victor D. Sanua, « Emigration of Sepharadic Jews from Egypt after the Arab-Israeli war », Los Muestros, N²5, Décembre 1996, pp. 17-21)

13105000 juifs Roumains ont émigré en Israël entre 1961-1980.

14 Ce dernier a créé par la suite l'active association des juifs soviétiques en Israël - le Forum sioniste des Juifs soviétiques - et est devenu le leader du parti russe Israel BaAliyah. Suite au succès de son parti aux élections de 1996, Nathan Charansky a obtenu le poste de Ministre de l'Industrie.

15 Selon Pierre Feuillie, environ 140000 immigrants auraient été logés dans ces logements abandonnés entre 1948 et 1949 in Pierre Feuillie, La politique d'immigration de l'Etat d'Israël (1948-1952), Nouvelles Presses Mondiales, Paris, 1953, p. 127.

16 Barnavi Elie, Une histoire moderne d'Israël, Flammarion, Paris, 1988, p. 121-122. 
En 1954, la politique d'aménagement du territoire prendra véritablement naissance avec «l'Opération du Bateau au village » où dès leur arrivée les olim sont emmenés dans les zones de développement. Globalement, cette logistique d'installation des immigrants est un succès - en termes de résorption de la vague migratoire - car entre 1948 et 1957 : plus de 400 villages ont vu le jour (en majorité des mochavim et kibboutzim $^{17}$ ), et la population rurale a pratiquement quadruplé évoluant de 110000 à 387000 personnes. ${ }^{18}$ Chaque nouvelle vague vient renforcer les structures établies et en créer de nouvelles en périphérie des préexistantes. Cette politique, qui a régi le plan d'aménagement du territoire israélien jusqu'à la fin des années soixante, bénéficiait à la fois du dynamisme créé par une industrie israélienne grandissante et du soutien de la diaspora (ses donations sont estimées entre 60 et 100 millions de dollars au cours des dix premières années auxquels il faut ajouter l'équivalent en achats de bons du Trésor israélien ${ }^{16}$ ).

Cette précarité de l'installation a probablement contribué à la faible participation migratoire des communautés occidentales à l'édification d'Israël ; mais c'est principalement dans le fort sentiment d'intégration de ces Juifs d'Occident à leurs sociétés que se trouvent les raisons de cette faiblesse. ${ }^{19}$ De 1952 à 1964 , seulement 2850 juifs de France, 2700 du Royaume-Uni, 8000 d'Argentine, 3650 des Etats-Unis et 500 du Canada ont immigré en Israël, alors que dans le même temps ils étaient 8300 à venir de l'Inde.

\section{Les premiers vacillements du concept d'intégration}

La poursuite du continuum migratoire, au cours des premières heures de l'Etat, va révéler la diversité du Peuple juif et donc voir la structuration de la mosaïque Israël. ${ }^{20}$ Même si l'édification de la société israélienne ne s'est pas réalisée à partir de

17 «Le kibboutz est une communauté fondée sur les principes de l'égalité et de l'autogestion. La production et la consommation sont collectives, tandis que dans le mochav, l'exploitation de la ferme familiale est incorporée dans les institutions coopératives du village. La formule mochav maintient l'unité de la cellule familiale» (Bensimon (D.) et Errera (E.), Op. Cit., pp. 42 et 53). En 1995, 123800 personnes vivent dans des kibboutzim et 176400 dans des mochavim.

18 Bensimon (D.), Errera (E.), Israéliens, des juifs et des Arabes, Ed. Complexe, Bruxelles (Historiques), 1989, p. 85.

19 Depuis longtemps les Juifs d'Europe ont cherché à s'émanciper notamment à travers la Haskala - mouvement des lumières juif développé par Moses Mendelshon dès le XVIII siècle - qui leur propose "d'être un homme au dehors et un Juif à la maison » (Yehouda (L.) Gordon, 1877). Si cette dynamique de l'émancipation a permis une importante restructuration du monde juif, elle a vu également l'antijudaïsme chrétien des sociétés d'accueil se muer en un antisémitisme moderne.

20 Nous employons ici le terme communément usité de «mosaïque » dans le sens où il figure une société pluriculturelle du fait de la multiplicité d'origines des migrants, même si cette métaphore est discutée actuellement par certains chercheurs. cf. Jonathan M. Schwartz, " "Mosaic" as metaphor of (Multi)cultural space », Migration, special issue Nordic countries, $23-24,1994$ 
la Déclaration d'indépendance, une indéniable accélération lui a été impulsée à cette période. Au cours des premières heures de l'Etat, cette construction s'est véritablement effectuée dans l'urgence. C'est certainement ainsi que cette «communauté de destin » a su se jouer, ipso facto, de cette question qui intriguait Vladimir Jankelevitch : "Comment les Juifs installés depuis si longtemps dans la langueur de l'absence, pouvaient échapper à l'angoisse des retrouvailles ? " ${ }^{21}$ Une réponse à cette absence d'angoisse résidait dans la puissance de la Histadrout - Confédération générale des travailleurs juifs créée en 1920 - Cette confédération avait su prendre en main la destinée du Ychouv en agissant tel un véritable gouvernement et comme l'a dit Hannah Arendt, «c'est ce qui explique le fait miraculeux qu'une simple proclamation de gouvernement autonome juif suffit en fin de compte à mettre en route un appareil d'Etat $\gg .{ }^{22}$ La promulgation de la Loi du Retour fut la décision majeure et hautement symbolique qui fut prise dans les premiers instants de l'Etat par ce gouvernement. Votée en 1950 par la Knesset, cette loi, pilier du droit du peuple juif, confère à tout juif le droit de s'établir en Israël et d'en acquérir automatiquement la nationalité.

Si dans un premier temps le jeune Etat a su gouverner et transiger sur le devenir du sionisme et par voie de conséquence, a su repousser cette angoisse des retrouvailles, l'agitation grandissante de la société va vite l'amener à affronter cette dernière. Le problème de l'édification nationale commençait en effet à connaître quelques vacillations et la question de l'intégration devenait de plus en plus saillante. Après le «succès » du Kibboutz Galouyoth (rassemblement des exilés), l'Etat devait maintenant relever le défi du Mizzug Galouyoth, la « fusion des exilés ». Dès la fin des années cinquante, la politique d'intégration des communautés a rapidement connu de graves tensions. L'insertion sociale des Orientaux - Mizrahim - s'est révélée difficile car la majorité d'entre eux ont été dirigés vers des zones de développement où les conditions de travail étaient d'une extrême rudesse et où leurs qualifications ne leur permettaient pas d'accéder à des emplois plus valorisants. ${ }^{23}$ Ces deux aspects cumulés ont poussé nombre d'entre eux à quitter leur lieu d'affectation pour trouver refuge dans les quartiers déshérités des villes de Jérusalem, de Tel Aviv, de Jaffa et de Haïfa. Dans cette dernière éclate en 1959, au sein du quartier de Wadi Salib peuplé de ces familles en perdition, la première révolte majeure des Orientaux contre l'establishment ashkénaze. Ces tensions n'étaient certes pas nouvelles, mais avec celles-ci, se dessinent les zones de fracture qui vont parcourir, tout au long des futures décennies, la société israélienne dont le centre, soutenu par les valeurs des $\mathrm{II}^{\circ}$ et $\mathrm{III}^{\circ}$ Aliya, sera fréquemment déstabilisé par les turbulences de sa périphérie mue par les espérances et les déceptions des Orientaux.

21 In Halter Marek, Un homme, un cri, Robert Laffont, Paris (Presses Pocket), 1991, p. 41

22 Arendt Hannah, "La paix ou l'armistice au Proche-Orient? », in Auschwitz et Jérusalem, Presses Pocket (Agora), $\mathrm{N}^{\circ} 129,1991$. Cet ouvrage regroupe certains des articles de l'auteur publiés entre 1941 et 1966.

23 Les Juifs d'Afrique du Nord constituaient $23 \%$ de la population des zones de développement contre $8 \%$ chez les originaires de Roumanie et $6 \%$ chez ceux de Pologne et de Russie (Chouraqui Andre, Op. Cit., p. 489). 
L'effervescence de la Guerre des Six-jours (1967) constitue une période d'apaisement des tensions d'autant plus que les Orientaux y font acte de loyauté et d'hérö̈sme. Même si cette guerre a "ressoudé » la société - rejudaïsation des valeurs israéliennes - et permis un rapprochement de cette dernière avec sa diaspora - un regain de l'aliya est remarquable ${ }^{24}$ (cf. Figure 1) -, les tensions n'en sont que temporairement repoussées. Fin 1970 , la situation s'embrase de nouveau avec l'émergence des "Panthères Noires", groupe de jeunes qui exprime haut et fort le désarroi des Orientaux et la discrimination qu'ils subissent. Un simple regard sur les déséquilibres sociaux de la population éclaire cette crise : une famille ashkénaze percevait en moyenne des revenus 1,4 fois plus élevés que ceux des Orientaux (différence d'autant plus forte que la taille des familles de ces derniers était plus élevée) ; le taux d'accession à l'enseignement supérieur était 6 à 7 fois plus important chez les Ashkénazes ; ceux-ci se trouvaient à la tête du pouvoir tandis que les Orientaux ne tenaient que des postes aux rôles secondaires. ${ }^{25}$ Il était d'autant plus difficile à la communauté orientale de faire valoir ses droits qu'elle ne disposait pas d'une élite qui pouvait réellement la représenter. La communauté nord-africaine notamment était arrivée sans son "leadership » qui avait préféré émigrer en France, aux Etats-Unis ou au Canada. ${ }^{26} \mathrm{Ce}$ climat de tensions associé au choc de la Guerre du Kippour et à la rudesse des années qui s'en suivirent ont conduit les Orientaux à exprimer leur mécontentent en amenant au pouvoir le Likoud ( parti de droite) en 1977. Cet avènement du parti de droite au pouvoir est lourd de conséquences morales pour les travaillistes, car ce n'était pas un vote pour la droite qu'avaient opéré les Orientaux, mais véritablement un vote contre la gauche. Cet acte contestataire exprimait à la fois :

- le rejet de la politique d'intégration établie par l'establishment ashkénaze qui niait et voulait gommer les particularismes culturels des olim Orientaux par peur d'une levantinisation de la société;

- la blessure que constituait pour les Orientaux, le fait de s'entendre répéter que le maintien de leur faible statut social provenait de leur manque de "positive predisposition » envers l'intégration comme l'envisageait S. N. Eisenstadt ${ }^{27}$;

- et sous un angle plus optimiste, ce vote annonçait «l'éclosion d'une assez large classe moyenne séfarade " qui a perçu, semble-t-il dans le Likoud, l'opportunité de poursuivre son ascension sociale bien plus facilement que sous l'étouffante protection du parti travailliste et ainsi pouvoir préserver son identité, révéler les particularismes des communautés orientales - Edot HaMizrah - .

24 C'est au cours des années qui ont suivi la Guerre de Kippour que s'est située la majorité des flux migratoires en provenance des communautés occidentales. Entre 1965-1979 ont été rassemblés $42 \%$ du flux français, $45 \%$ du flux britannique et $55 \%$ du flux américain qu'a connu Israël entre 1948 et 1993.

25 Citation de Sammy Smooha faite par Uri Ram dans son ouvrage : The Changing agenda of Israeli sociology. Theory, ideology and identity, State university of New York Press, New York (SUNY series in Israeli Studies), 1995, p. 99.

26 Barnavi Elie, Op. Cit., p. 135

27 Cf. Ram Uri, Op. Cit., p. 39. S. N. Eisenstadt est l'auteur de très nombreux travaux sur la politique d'intégration des nouveaux immigrants en Israël en termes de sociologie. 
A travers cette dynamique de revendication sur une réelle base ethnique, un "Second Israël », oriental, devenait visible et l'élite ashkénaze de la société ne pouvait qu'intégrer cette nouvelle image d'Israël, antithèse de celle que s'étaient construit les pères fondateurs. Comme l'a titré, plus tard, S. N. Eisenstadt dans l'un de ses écrits - The problem of Edot - Not just transitory -, la société israélienne à la fin des années soixante-dix s'affirmait bel et bien comme une société pluri-ethnique. ${ }^{28}$

\section{LES ANNÉES 1980 : L'AUBE D'UN NOUVEAU CONTEXTE MIGRATOIRE}

Cette décennie est celle d'une réelle recomposition de la société israélienne en termes démographiques. Le changement est d'autant plus ressenti qu'à la fin des années soixante-dix s'est déjà opérée une importante révolution avec la perte du pouvoir par les travaillistes. L'héritage du sionisme pionnier qu'incarnait l'idéal socialiste semble ainsi ne plus être partie intégrante du patrimoine culturel israélien. La distanciation des relations entre la diaspora et Israël, affirmée par la faible attractivité de l'Etat, contribue à la désuétude du sionisme ou, plus justement, à l'affirmation de l'entrée d'Israël dans une ère post-sioniste.

\section{Une rupture dans la dynamique migratoire}

Le sentiment, le plus perceptible à la lecture de la courbe migratoire israélienne au cours des années quatre-vingt, est bien celui d'une fin de l'ère des grandes aliyoth. Le flux d'entrées pour l'entière décennie quatre-vingt arrive juste à dépasser ceux rassemblés au cours de la seconde moitié de la décennie soixante-dix. Seules 153000 entrées sont comptabilisées entre 1980 et 1989 contre 124000 entre 1975 et 1979 (cf. Figure 1). Il faut toutefois souligner que le droit d'émigrer étant refusé à la communauté soviétique, Israël s'est trouvé "amputé » d'un des foyers les plus réceptifs à son appel. La cause de cette interdiction de sortie est liée principalement à la condamnation par les Etats-Unis de l'intervention soviétique en Afghanistan. Ces années-là voient d'ailleurs une nette rupture du système « Relations Est-Ouest/Globalisation des échanges/Liberté de circulation », installé depuis la fin des années soixante, puisqu'entre 1979 et 1980 les échanges commerciaux entre les EtatsUnis et l'URSS, et l'émigration juive d'ex-URSS, chutent simultanément et corrélativement de moitié. Le volume d'échanges passe de 2,8 millions à 1,5 millions de roubles et le flux d'émigration juive est passé de 50000 à 20000 personnes.

Au-delà de ces freins extra-communautaires à la migration, Israël entre, de par cette rupture du continuum, dans une phase de redéfinition, de remise en cause de sa centralité. Peut-être, dans une phase où ce jeune Etat apprend à s'affirmer, s'émanciper

28 Eisenstadt (S.N.), The development of the ethnic problem in israeli society. Observations and suggestions for research, The Jerusalem Institute for Israel Studies, Jerusalem, 1986. 
Tableau 1 : Population juive selon le lieu de naissance entre 1948 et 1995

\begin{tabular}{|c|c|c|c|c|c|c|}
\hline \multicolumn{1}{|c|}{ Années } & 8 XI 1948 & 22 V 1961 & 19 V 1972 & 4 VI 1983 & 31 XII 1990 & 31 XII 1995 \\
\hline $\begin{array}{l}\text { Population totale } \\
\text { d'Israël }\end{array}$ & 872700 & 2179500 & 3147700 & 4037600 & 4821700 & 5619000 \\
$\begin{array}{l}\text { Population juive } \\
\text { dont: }\end{array}$ & 716700 & 1932400 & 2686700 & 3350000 & 3946700 & 4549500 \\
$\begin{array}{c}\text { Population née } \\
\text { en Israël }\end{array}$ & 253700 & 730400 & 1272300 & 1927900 & 2442900 & 2784300 \\
$\begin{array}{c}\text { Population née } \\
\text { à l'étranger }\end{array}$ & 463000 & 1201900 & 1414400 & 1422100 & 1503700 & 1765200 \\
\hline
\end{tabular}

Source : Statistical Abstract of Israel 1995, $N^{\circ} 46,1996$, p. 98 et Israel in figures (1960-1995), reprint from Statistical Abstract of Israel 1996, $N^{\circ} 47$, p. 3, CBS, Jerusalem.

un siècle après que les Biluhim aient posé leur pied sur le sol de Palestine. Au cours de ces deux décennies, l'Etat d'Israël voit la structure de sa population se modifier, la population née dans le pays devenant prédominante par rapport à celle née à l'étranger, jusque-là toujours majoritaire (cf. Tableau 1). En 1990, la part de population née en Israël constitue $61,9 \%$ de la population juive totale alors qu'en 1972, celle-ci ne regroupait que $47,3 \%$ et n'avait atteint l'équilibre qu'au début des années quatre-vingt. Durant cette période, l'origine des parents de ces enfants nés en Israël a évolué : en 1972 seulement $8,5 \%$ des sabarim $^{29}$ avaient un père né aussi en Israël ; en 1983 et en 1994, cette part monte respectivement, à $15,9 \%$ et $24,4 \%$. Par cette recomposition structurelle, l'image d'Israël en tant que terre de retour s'estompe peu à peu.

La rupture du continuum migratoire s'impose d'autant plus à l'esprit que l'émigration - Yerida -, antithèse du sionisme, vient témoigner de la faible attractivité d'Israël. ${ }^{30}$ Au cours des années 80 , les départs se font de plus en plus nombreux et vont jusqu'à mettre en péril la balance migratoire israélienne. Sur la période allant de 1980 à 1989 , le solde net de l'immigration s'est élevé à 22000 seulement ${ }^{31}$ (le solde migratoire annuel a été de -4700 et -4800 en 1985, 1986). Fin 1985, on comptait déjà près de 260000 Israéliens résidant à l'étranger depuis plus de 4 ans. ${ }^{32}$ Cette population expatriée s'élève à 338000 en 1992 dont plus de la moitié (183 900) a quitté le pays après 1973. L'Etat d'Israël, à travers le programme

29 Le terme de sabarim (sg. sabra) définit les personnes nées en Israël.

30 Mot hébreu signifiant « descente » utilisé en opposition à la «montée» qu'est l'aliyah. Le terme de yored (émigrant) est également utilisé en opposition à celui d'oleh (immigrant).

31 Coubage (Y.), "Contrastes démographiques en Israël : tendances récentes », Population, $\mathrm{N}^{\circ} 3,1993$, p. 754.

32 Cf. I'article de Sergio Della Pergola dans ce numéro ainsi que : Eitan Sabatello, "Evaluation de l'émigration d'Israël : mesures actuelles et perspectives ", in Les migrations internationales, problèmes de mesure, évolutions récentes et efficacité des politiques (Séminaire de Calabre 8-10 septembre 1986), AIDELF, N³, 1988 ; Central Bureau of Statistics, "Indicators of the number of Israeli residents abroad 1992 », reprint from the supplement to the monthly bulletin, $\mathrm{N}^{\circ} 6$, Jerusalem, 1994. 
"Returning Israelis » financé par le ministère de l'Intégration, tente aujourd'hui d'inciter au retour ses expatriés en leur proposant une aide à la « réintégration » ou tout du moins veille à ne pas perdre le contact avec eux. Les résultats de ce programme sur les cinq dernières années indiquent une progression des retours puisque le flux est passé de 8000 à 14000 retours entre 1991 et $1995 .^{33}$

\section{L'expression d'un pluriculturalisme israélien}

La recomposition du continuum migratoire, qui s'est opérée au cours des années soixante-dix-quatre-vingt, a eu d'importantes conséquences identitaires pour Israël. La manifestation d'un «Second Israël » - fruit des frustrations des séfarades vis-à-vis du «Premier Israël », ashkénaze -, où ont su se fédérer les Orientaux, en a été l'une des principales expressions. Paradoxalement, cette ethnicisation, que l'on aurait pu croire désintégratrice de par sa dynamique séparatiste, se révèle être au contraire comme l'a décrit $\mathrm{M}$. Abitbol, "toute tournée vers le centre puisqu'elle est vécue le plus souvent comme un moyen instrumental destiné à forcer l'accès du centre et de ses symboles $\gg .{ }^{34}$ Cette conjoncture apparaît donc comme productrice d'une création endogène signifiant à la fois une valeur d'intégration de la mosaïque Israël ainsi qu'une réactualisation des rôles et relations au sein de cette société pluriculturelle.

Un simple regard sur la composition de la population israélienne à travers le prisme de son histoire migratoire montre que ce pluralisme identitaire ne pouvait générer qu'une synthèse d'une grande complexité. En effet, c'est dans plus d'une soixantaine de pays et de bien plus encore de sous-espaces aux identités variées et marquées que la population immigrée d'Israël trouve ses origines (cf. Carte 1). Ce flux migratoire ne rassemble pas moins de 1834000 personnes sur la période allant de la création de l'Etat à l'aube de la grande vague migratoire d'ex-URSS en 1990. De plus, l'affaiblissement des flux migratoires au cours des années quatre-vingt fut l'instant propice où la population a exprimé le désir de discuter l'unicité d'Israël.

Cette société a d'une certaine manière été "revivifiée » par l'expression ethnique et a vu s'opérer une symbiose entre Orientaux et Occidentaux. Une rencontre de ces deux mondes a su s'établir sur une fracture néanmoins toujours sous-jacente à travers les générations de sabarim. Cette «synthèse sabraïque », caractérisée par la

33 En 1992, 10500 retours (dont 5000 bénéficiaires du programme), en 1993, 13000 (7 000), en 1994, 14000 (8 000). Notons qu'un résident de retour est un israélien agé de 17 ans et plus qui est resté une période minimun de 2 ans à l'étranger en dehors d'un contrat au service de l'Etat d'Israel ou d'un autre de ses services officiels. Source : Immigrant absorption, situation, challenges and goals, Ministry of Immigrant Absorption, Planning and Research division, Jerusalem, april 1996, p. 58.

34 Cf. Abitbol (M.), «La mémoire occultée et retrouvée : Juifs d'Orient et de Méditerranée en Israël » in Heymann (FI.) (ed.), Les nouveaux enjeux de l'historiographie israélienne, Lettre d'information du Centre de recherche français de Jérusalem, décembre 1995, $\mathrm{n}^{\circ} 12$, pp. 41 49. Les lettres d'information du CRFJ éditées par Florence Heymann (depuis 1982) constituent une source d'informations en langue française sur la société israélienne d'une très haute qualité scientifique. 
PAYS DE NAISSANCE DES IMMIGRANTS ARRIVES EN ISRAËL ENTRE 1948 ET 1993

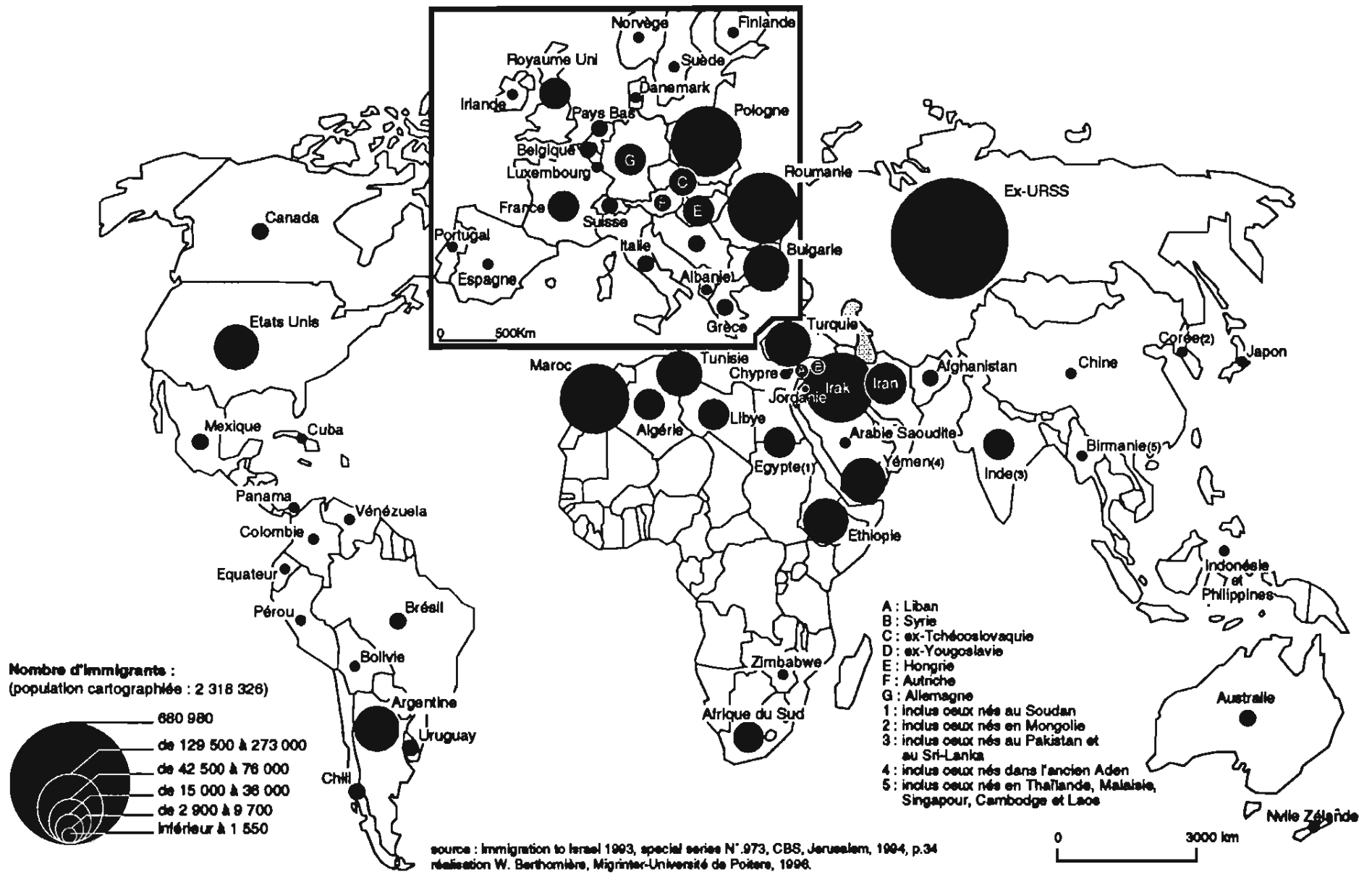


complexité de ses racines, a été facilitée par le fait que les Orientaux ont poursuivi leur processus d'ascension sociale. Des indicateurs comme la croissance de la part des étudiants d'origine orientale (sur la période 1960-1980, leur nombre absolu est passé de 1500 à $16000^{35}$ ), la réduction de l'écart entre les taux de natalité des deux groupes, la multiplication des «mariages mixtes " chez les nouvelles générations, l'accession à des postes de décision tant au niveau local que national et l'émergence d'une intelligentsia séfarade sont les garants de cette synthèse et de son renforcement au sein des futures générations de sabarim.

Cette dynamique au sein de la société israélienne est parfaitement reflétée par la nouvelle école de sociologie israélienne - avec Baruch Kimmerling, Uri Ram et Sammy Smooha en tête - qui a "revisité » le schéma social élaboré par ses prédécesseurs. Comme le souligne avec justesse Danielle Storper Perez, le schéma de pensée en termes d'approche du phénomène migratoire a vu se substituer à la perspective assimilationniste une orientation pluraliste. ${ }^{36}$ La société est ainsi envisagée, selon S. Smooha, comme une " composante de fragments diversifiés culturellement et inégaux sur le plan socio-économique ». Cette "nouvelle nation » se conçoit comme un tout où l'on discerne un centre et des périphéries aux anciennetés et importances variées mais toujours reliés entre eux par des liens plus ou moins serrés, que vont devoir renforcer, et peut être diversifier dans le futur, les vagues migratoires d'Ethiopie et d'ex-Union Soviétique.

\section{LES VAGUES MIGRATOIRES ETHIOPIENNE ET EX- SOVIETIQUE OU UN CONTINUUM MIGRATOIRE REVIVIFIE?}

L'entrée dans la décennie quatre-vingt-dix marque pour Israël une nouvelle transition dans le continuum migratoire. Israël renoue avec une immigration qui n'est pas sans rappeler celle des premières heures de l'Etat et par voie de conséquence avec des préoccupations - accueil et intégration des immigrants - qui questionnent à nouveau son identité. Cette question de l'interaction de cette immigration dans l'édification de l'ethos national est d'autant plus prééminente que l'actuelle configuration migratoire est totalement différente. Il s'agit maintenant non pas d'une grande vague migratoire composée de multiples identités mais d'une vague massive pourvue de deux identités dominantes. De plus, cette migration est, pour la communauté ex-soviétique, nettement moins empreinte des sentiments sionistes et religieux que les précédentes aliyoth.

35 Bensimon (D.), Errera (E.), Op. Cit., p. 176

36 Storper Perez (D.), "L'impérialisme de la fonction : l'approche fonctionnaliste du phénomène migratoire " in Heymann (Fl.) (ed.), Identités et cultures. La recherche israélienne au miroir du Centre de Recherche Français de Jérusalem, Lettre d'information du CRFJ, Jérusalem, N 13, octobre 1996, p. 53. 
La lecture de la figure 2 confirme une réelle mutation du continuum migratoire. Il ne s'agit plus ici d'une soixantaine de flux d'origines différentes en mouvement vers cette centralité que "concrétisait alors Israël », mais de deux flux particulièrement élevés par rapport à ceux en provenance des grands foyers de cristallisation de la diaspora (Etats-Unis, France, Royaume Uni, Argentine et Afrique du Sud). ${ }^{37}$

\section{Figure 2}

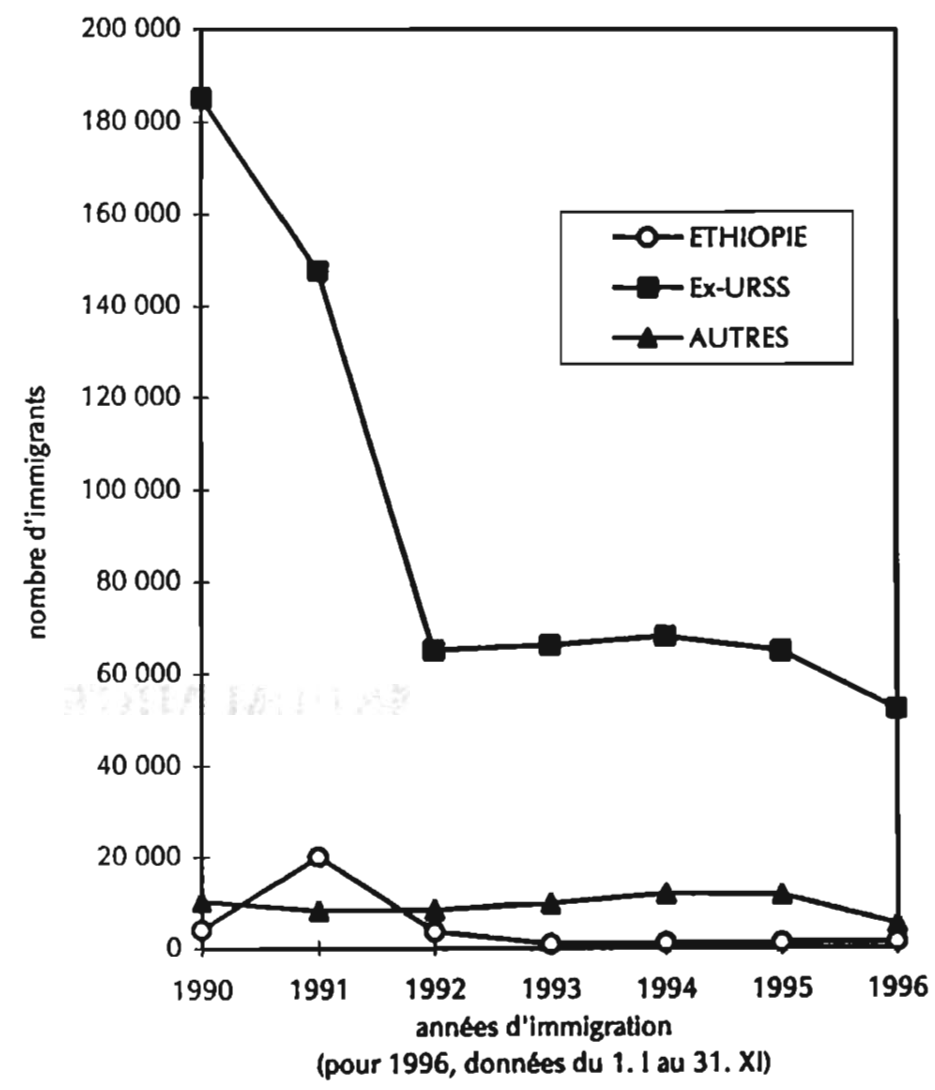

Source : Central Bureau of Statistics, Jerusalem.

37 En 1990, seuls huit pays regroupaient plus de 100000 membres de la diaspora : 5515000 aux Etats Unis, 1150000 en ex-URSS, 530000 en France, 315000 en Grande Bretagne, 310000 au Canada, 215000 en Argentine, 114000 en Afrique du Sud et 100000 au Brésil. Notons que pour les Etats Unis, cette estimation concerne les personnes qui se définissent comme juif mais si l'on intègre les personnes d'origine juive pratiquant une autre religion et les membres non juifs des familles mixtes, la communauté s'élève alors à 819000 . (source : Bensimon (D.), Les Juifs dans le monde au tournant du XXI siècle, Albin Michel (Présences du Judaïsme), Paris, 1994, p. 15 et p. 45) 
Entre 1990 et 1996, Israël a accueilli près de 750000 personnes dont les seuls flux d'ex-URSS et d'Ethiopie constituent les neuf dixièmes. ${ }^{38}$ Les autres flux migratoires en direction d'Israël sont d'une réelle faiblesse en comparaison des deux précédents. Uls ont rassemblé en moyenne environ 10000 immigrants par an sur cette période, soit un niveau d'entrée équivalent aux périodes antérieures. La vague massive en provenance d'ex-URSS et d'Ethiopie, notamment en 1990 et 1991 avec respectivement 189348 et 167853 personnes, n'a donc pas été un frein à l'immigration pour les autres foyers de la diaspora. Cette vague migratoire a globalement regroupé en sept ans près d'un tiers de l'immigration qu'a connu Israël depuis 1948 (environ 2580000 personnes).

\section{Les Beta Israel ou le transfert d'une communauté}

Le terme de Beta Israel désigne la communauté juive éthiopienne plus connue en Europe sous le nom de Falasha. Cette dernière dénomination a tendance à disparaître du vocabulaire israélien car elle est avant tout offensante pour cette communauté et décrit cette dernière dans son contexte éthiopien et non israélien. Le caractère péjoratif provient du fait que le nom «falasha» dérive d'un terme guèze qui exprime l'exilé, l'étranger, le vagabond. Les dénominations, telles que « Juifs Ethiopiens » et «Beta Israel », lui ont donc été préférées. ${ }^{39}$

L'histoire du transfert de cette communauté juive vers Israël est pour l'essentiel celle des deux «opérations de sauvetage » nommées Moïse et Salomon. «L'opération Moïse » a permis la venue de 7000 Beta Israel entre les mois de novembre 1984 et janvier 1985. Quelques 6000 Ethiopiens avaient toutefois précédé cette opération et 11000 autres l'avait suivie jusqu'au déclenchement de "l'opération Salomon ». Ce pont aérien réalisé en 36 heures au cours du mois de mai 1991 a permis le transfert de 14300 Ethiopiens. Depuis ces opérations, l'immigration éthiopienne se poursuit mais à un rythme moindre. En moyenne, le flux annuel s'élève à 1500 entrées. Les flux les plus récents sont composés en grande partie de Falash Mura $^{40}$, personnes converties au christianisme se réclamant de descendants des Beta Israel, qui émettent aujourd'hui le désir de rejoindre Israël. Ce désir d'émigration doit être compris comme une volonté de regroupement familial : en effet, dans nombre de familles éthiopiennes, des membres ont conservé une pratique du judaïsme, tandis que d'autres se sont convertis. Les Falash Mura entrent, pour la plupart, dans le cadre de la

38 Le lecteur pourra consulter, pour plus d'informations statistiques sur l'immigration notamment, le serveur du Central Bureau of Statistics sur internet : «http://www.cbs.gov.il».

39 Pour des informations sur l'histoire de cette communauté, voir les travaux de Steven Kaplan. Le site Internet sur les Juifs d'Ethiopie comporte également de nombreuses informations sur ce thème ainsi que des cartes de localisation de cette communauté en Ethiopie (http://www.cais.com/nacoej/). En ce qui concerne la désignation du groupe, cf. WEIL Sh., «Collective designations and collective identity among Ethiopian Jews», Israel Social Science Research, Vol. 10, No 2, 1995, pp. 25-40.

40 Pour une information concise sur les Falash Mura, cf. Kaplan (S.), «Falasha-Christians : a brief history », Midstream, vol. 39, $\mathrm{N}^{\circ} 1,1993$, pp. 20-21. 
« loi d'entrée " ${ }^{41}$ selon le critère de « réunification familiale » et non dans le cadre de la « loi du retour ».

La communauté éthiopienne regroupe actuellement 57000 personnes, dont 10500 sont nées en Israël. Cette population se caractérise par son extrême jeunesse : la classe d'âge 0-18 ans rassemble la moitié de la communauté, alors que cette classe ne constitue qu'un quart de la communauté ex-soviétique, et deux tiers de la population juive d'Israël.

La répartition spatiale des Ethiopiens arrivés lors de l'opération Salomon, ou depuis celle-ci, est très largement influencée par la politique d'aide à l'acquisition de logements mise en place par l'Etat. Depuis mai 1993, environ 3500 familles ont bénéficié de prêts subventionnés par l'Etat. ${ }^{42}$ Diverses zones d'acquisition immobilière ont été définies (environ 50 villes), essentiellement dans la région Centre, et l'actuelle répartition des Ethiopiens en Israël en découle (cf. Carte 2). Cette politique à la fois volontariste et libérale, puisqu'elle offre aux Ethiopiens un vaste choix de lieux de résidence, a été motivée par les enseignements de l'intégration spatiale des personnes arrivées lors de "l'opération Moïse ». A l'époque, la politique israélienne de répartition de cette population s'était heurtée à une vision de l'espace totalement différente chez les Ethiopiens. Ainsi, certaines localités avaient vu leurs logements d'accueil rester désespérément vides tandis que d'autres s'inquiétaient devant la transformation de ces lieux d'accueil en véritables "espaces-ghettos ". ${ }^{43}$ Lors de «l'opération Salomon », la reproduction de tels comportements a en partie influencé la décision d'enlever de la liste des villes offrant des prêts immobiliers, les localités de Lod, Yavne et Kiryat Gat, très attractives, qui accroissaient le risque de «ghettoösation».

Par une politique interventionniste et l'entrée progressive des Ethiopiens dans la société israélienne, les conditions de logement de cette communauté se sont améliorées au cours des quatre dernières années. Les 22 sites de caravanes, installés pour pallier le manque de logements (sites qui n'étaient pas sans rappeler les Ma'abaroth des premières heures de l'Etat), ont commencé à se dépeupler. Si en 1992, plus de 5000 familles et 1700 célibataires ne possédaient pas de résidence permanente (plus des deux tiers étaient dans des sites de caravanes), fin 1995, on ne comptait plus que 550 familles en attente d'un logement. Les célibataires se maintiennent toutefois à 900 , dont 200 résident dans des sites dits « de jeunesse ».

Ce changement de situation par rapport à la résidence a été précédé, pour la majorité des Ethiopiens, par l'obtention d'un emploi. Le taux d'emploi chez les

41 Loi qui régit l'entrée et le statut des immigrants non-juifs.

42 La plus grande partie de ces familles ont sollicité des prêts d'environ 550000 francs dont $85 \%$ à $90 \%$ constituent une sorte de subvention de l'Etat tandis que le solde (les 15 à $20 \%$ restants) doit être remboursé par mensualités d'environ 250 francs.

$43 \mathrm{Cf}$. Rosen (H.), "A government anthropologist among the Ethiopian Jews in Israel », Israel Social Science Research, Vol. 10, No 2, 1995. 
Carte 2

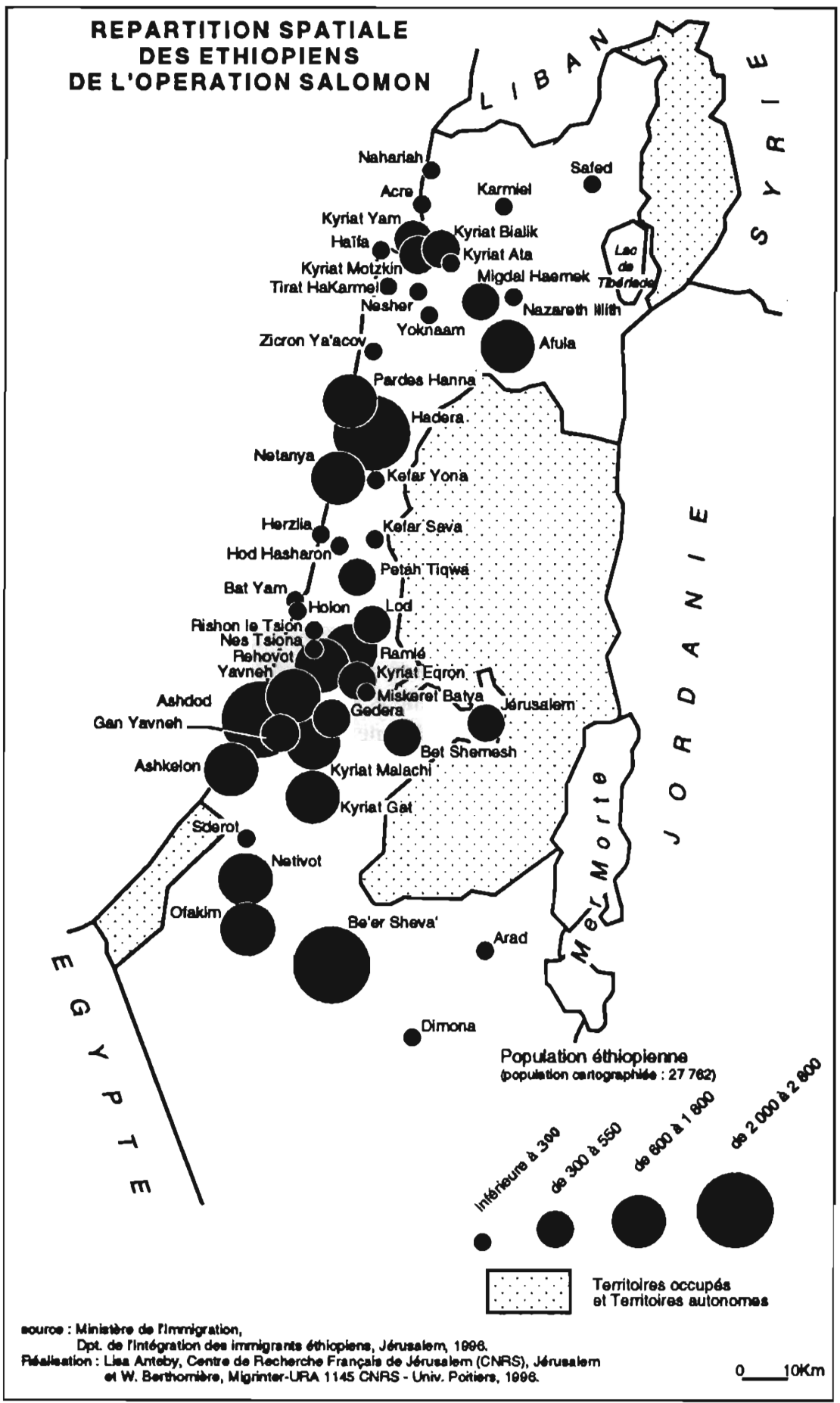

REMI 1996 (12) 3 pp. 33-60 
résidents de caravanes est passé, fin 1992, de $40 \%$ à $80-85 \%$ fin 1995. Sur cette même période le budget du ministère de l'Intégration consacré à l'emploi aura lui aussi doublé pour s'élever à près de 7 millions de francs. Globalement, la communauté éthiopienne à travers les différentes aides gouvernementales ainsi que par ses propres initiatives, acquiert une plus grande mobilité sociale. Cette "venue à communauté " s'opère par les voies classiques de l'intégration en Israël, en premier lieu par l'étude de l'hébreu, puis suivant les classes d'âges, par le système éducatif et l'armée pour les plus jeunes, et par les cours de formation professionnelle pour les plus âgés. Dans cette dynamique d'emploi, environ une trentaine de petites entreprises ont été créées au cours des dernières années. Toutefois, même si cette communauté est parvenue à un taux d'emploi conforme à la moyenne nationale et possède maintenant un représentant à la Knesset, nombreux sont ceux qui s'inquiètent pour elle. Certains confient que ces derniers ne sont pas faits pour "le monde du travail israélien infesté de requins", d'autres les qualifient de "génération du désert » et soulignent le "défaitisme » des pouvoirs publics. L'association de défense des Juifs éthiopiens, quant à elle, ne manque pas de souligner que cette communauté constitue près de $10 \%$ des quelques 700000 israéliens vivant en dessous le seuil de pauvreté. ${ }^{44}$

Autant de points qui amènent à conclure que l'intégration n'est pas uniquement une affaire de temps, même si celui-ci s'avère être un paramètre fondamental dans ce processus de "venue à communauté ». La dimension dite «d'intégration sociale », qui constitue le dernier élément de la "bonne intégration » telle qu'elle a été définie en conclusion du récent rapport sur les nouveaux immigrants ${ }^{45}$, est très certainement la dimension nécessitant le plus de temps pour s'accomplir dans le processus multidimensionnel qu'est l'intégration. Esther Benita et Gila Noam nous confirment que cette dimension chez les Ethiopiens est, comme chez d'autres communautés immigrantes de par le monde, tributaire de l'acquisition de conditions de vie décentes (logements, emplois) et d'une connaissance suffisante de la langue du pays d'accueil. ${ }^{46}$ L'ouverture sur la société en est ainsi facilitée et la tentation d'un repli identitaire, voire d'une "ghettoïsation", s'en trouve repoussée. Dans leurs enquêtes menées au sein de différentes agglomérations, notamment Afula, Netanya et Kiryat Gat, elles ont montré que la possession d'un emploi et la durée de résidence en Israël sont autant de facteurs qui contribuent à ne plus faire de la résidence auprès d'autres Ethiopiens, une priorité. La durée de résidence semble également favoriser la rencontre des enfants de cette communauté avec les jeunes sabarim notamment, puisqu'avec le temps seulement $10 \%$ des familles interrogées affirment que leurs enfants ne jouent qu'avec d'autres jeunes d'origine éthiopienne. Cette rencontre des fils et des filles d'Israël sera certainement le garant de l'intégration

44 Cf. article de Hoch (D.), The Jerusalem Post/Edition française internationale - semaine du 16 au 22 octobre 1996.

45 Immigrant absorption, situation, challenges and goals, Ministry of Immigrant Absorption, Planning and Research division, Jerusalem, april 1996, p. 62.

46 Benita (E.) et Noam (G.), Absorption of Ethiopian Immigrants : Selected Findings, from Local Surveys, Israel Social Science Research, Vol. 10, N² 1995. 
sociale de cette jeune génération d'origine éthiopienne, ne serait-ce par le simple fait qu'elle repousse, espérons-le, d'éventuels sentiments racistes.

\section{L'immigration d'ex-URSS, un flux d'une réelle amplitude}

Les dernières données statistiques indiquent que depuis 1990, 647900 personnes en provenance d'ex-URSS sont entrées en Israël ${ }^{47}$. Cette statistique révèle à la fois l'importance de cette immigration qui place Israël parmi les premiers pays d'immigration au monde -surtout en proportion de sa population- et la centralité du flux migratoire en provenance d'ex-URSS puisqu'il constitue $86,5 \%$ du flux global. On notera que l'immigration en provenance d'ex-URSS pour la seule année 1990 (185 227) fut plus importante que le flux global de la décennie quatre-vingt (153 833) et que par ailleurs cette vague migratoire a rassemblé en sept ans, l'équivalent de l'immigration des années 1965 à 1989 (dont près d'un tiers provenait d'ailleurs d'Union soviétique). En outre, pour le seul mois de décembre 1990, ce flux a rassemblé un nombre équivalent à l'ensemble du flux français entre 1919 et 1996, soit environ 35000 personnes.

Si la population israélienne a pu exprimer sa joie et affirmer l'importance du rôle d'Israël lors des opérations Moïse et Salomon, sa réaction n'a pas été tout à fait la même avec l'aliya d'ex-URSS. La population israélienne et celle des nouveaux immigrants se sont quelque peu heurtées. Cette dernière, munie d'un bagage intellectuel conséquent, n'appréciait guère que ses problèmes d'intégration ne soient pas plus efficacement résolus, tandis que les vétérans semblaient agacés par l'arrogance des ex-soviétiques. C'est donc dans une ambiance d'une réelle étrangeté qu'a débuté l'entrée de ces milliers d'olim en Israël. Les ingénieurs russes qui se retrouvaient à nettoyer les rues, s'entendaient dire par la population, pour seule consolation, qu'elle aussi avait fait de «sales boulots». La population locale à son tour, restait bouche bée devant le défilé en uniforme et médailles au complet des anciens de l'Armée Rouge à Kiryat Gat aux portes du Neguev. Pour les Israéliens, cette vague migratoire était à la fois merveilleuse et effrayante.

Ces milliers d'immigrants se sont répartis dans les localités israéliennes de leur choix dans le cadre de "l'intégration directe ». Cette politique du libre choix de résidence allait-elle pour autant générer la mise en place de filières migratoires au sens d'une répartition communautaire (liée au lieu d'origine en ex-URSS) ? L'inscription spatiale des migrants ne semble pas attester de la présence de réelles filières migratoires facilement identifiables. ${ }^{48}$ Les nouveaux arrivants se sont orientés

47 Donnée entre le 1.I.1990 et le 31.XI.1996.

48 Les données et résultats présentés ci-dessous sont issus d'une extraction spéciale concernant les immigrants d'ex-URSS arrivés en Israël entre le 01/01/1990 et le 31/12/93 soit environ 460000 personnes (plus des $2 / 3$ de la communauté présente dans le pays en septembre 1996). Les données sont ventilées suivant la ville de départ en ex-URSS et la ville de première résidence en Israël. Les migrations internes, qui ont pu intervenir, depuis faussent quelque peu l'analyse mais dans une faible proportion toutefois car notre échantillon regroupe la grande aliyah (1990-93) et seulement un faible pourcentage de nouveaux arrivants par rapport au volume migratoire global s'est redéplacé.

REMI 1996 (12) 3 pp. 33-60 
essentiellement vers les principales agglomérations d'Israël : Tel Aviv, Haïfa, Jérusalem et Beer Sheva (cf. carte 3). Près de la moitié des nouveaux immigrants d'exURSS est regroupée dans les dix premières localités d'Israël en 1993, soit plus de 200000 personnes motivées par leur forte mentalité urbaine et par l'idée que ces localités constituent les plus importants bassins d'emplois du pays. ${ }^{49} \mathrm{Par}$ comparaison, ces choix de résidence sont globalement identiques à ceux des migrants soviétiques arrivés entre 1948 à $1983 .^{50}$

Tel Aviv s'affirme comme le premier espace d'accueil en concurrence avec Haïfa. Si l'on observe la situation à travers les flux issus des principaux centres de la communauté en ex-URSS, Haïfa apparaît être le premier choix de résidence de huit communautés sur les dix-sept ici analysées et Jérusalem n'est privilégiée que par trois de celles-ci. Cette ville mythique n'a attiré sur la période considérée que les communautés de Moscou, de Leningrad et de Tallinn, et n'apparaît pas parmi les six principales résidences des communautés issues de Vilnius, Bakou ou bien encore Kishinev. Pour cette dernière communauté, Haïfa a reçu cinq fois plus de migrants que Jérusalem, respectivement 1460 et 270 personnes.

Outre ces premiers constats, cette répartition affirme avant tout l'attrait des grandes villes d'Israël et révèle la présence d'espaces d'absorption secondaires constitués par leurs aires métropolitaines. Ce second espace d'intégration est composé principalement par : Rishon le Zion, Petah Tiqwa, Bat Yam, Holon, Ashdod, Ashqelon ou bien encore Netanya. Ces villes se situent en majorité dans l'aire métropolitaine de Tel Aviv comme elle a été définie pour le recensement effectué en $1995 .^{51}$

Malgré cette répartition qui semble en conformité avec la structure urbaine israélienne, quelques caractéristiques « ethniques » apparaissent :

- près des deux tiers de la communauté russe de Jérusalem sont composés des migrants de Moscou et de Leningrad alors qu'ils ne constituent que $40 \%$ du flux global issu de la Russie. Cette prédilection provient essentiellement de l'attrait de cette ville mythique sur ces migrants issus des deux grandes agglomérations russes, notamment pour l'intelligentsia ;

- la communauté en provenance de Derbent (Daghestan) s'est dirigée en majorité vers Hadera (30\% du flux global avec 1250 personnes), soit loin devant la seconde ville d'accueil Beer Sheva (600 personnes). Les nouveaux immigrants provenant de Derbent composent d'ailleurs près d'un tiers de la communauté russe de cette ville (les communautés de Moscou et Leningrad n'en composant que $15 \%$ ) ;

49 Cf. Berthomière (W.), L'immigration des Juifs d'ex-URSS : Un nouveau défi pour Israël ?, REMI, vol. 11, N 3, 1995.

$50 \mathrm{Cf}$. Efrat (E.), Geographical distribution of the Soviet-jewish new immigrants in Israel, Geojoumal, N ${ }^{\circ} 24-4,1991$

51 Lors du dernier recensement de population, les services statistiques ont opéré quelques modifications qui ont vu le statut de conurbation être remplacé par celui d'aire métropolitaine dans le cas de Haïfa et Tel Aviv. Pour cette dernière, l'aire métropolitaine s'étend du nord de Netanya au sud d'Ashdod. 


\section{Carte 3}

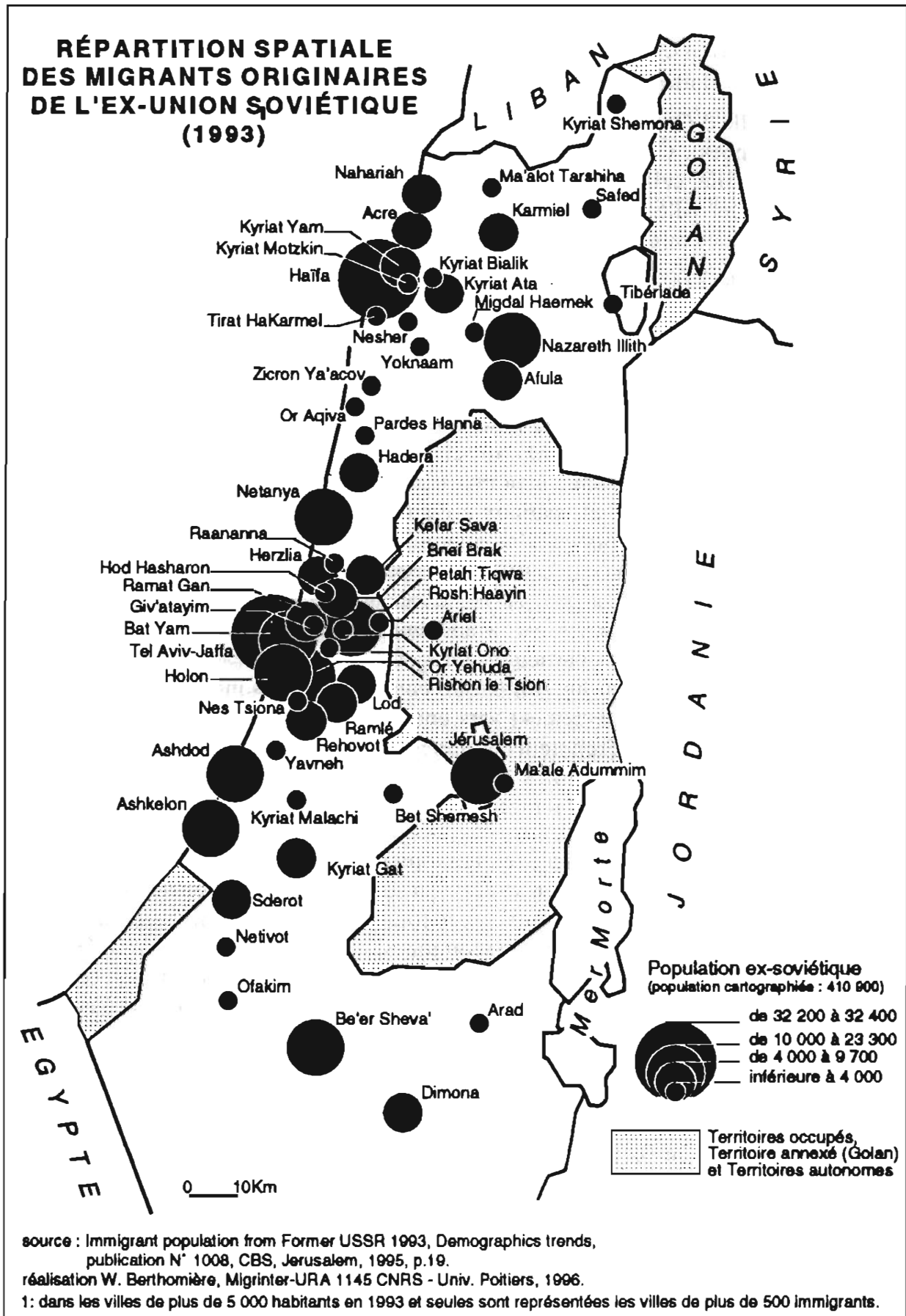

REMI 1996 (12) 3 pp. 33-60 
- au sein de la communauté biélorusse, près de six fois plus de migrants de Gomel que de Minsk se sont orientés vers Nahariah (respectivement 600 et 100 personnes). Ces deux communautés représentent toutefois près de la moitié de la communauté biélorusse de cette localité située à la frontière septentrionale de l'aire métropolitaine de Haïfa ;

- la ville d'Or Yehuda regroupe plus de $15 \%$ de la communauté de Samarkand (soit 880 personnes). Cette dernière compose ainsi les deux tiers de la communauté ouzbèque de la ville, celle de Tachkent n'en constituant que $15 \%$. Ainsi, Or Yehuda et Tel Aviv (première ville d'installation avec 1450 personnes) rassemblent à elles deux près de la moitié des migrants de Samarkand;

- plus de 2000 membres de la communauté de Kharkov se sont rassemblés à Haïfa soit trois fois plus que dans leur seconde ville d'accueil, Jérusalem (avec 640 immigrants).

Au cours des entretiens que nous avons réalisés auprès des nouveaux entrepreneurs d'ex-URSS en Israël, nous avons interrogé ces derniers, originaires des diverses ex-républiques, sur l'existence d'éventuelles filières et sous-filières migratoires. Dans leur très grande majorité, les interviewés ont affirmé que pour eux de tels comportements sociaux et spatiaux n'étaient pas si prégnants. Certains révèlent néanmoins des comportements qui s'en approchent comme le fait que les Odessites se dirigent avant tout vers Haïfa car le site leur rappelle celui d'Odessa tandis que ceux de St Pétersbourg privilégieraient pour la même raison la ville de Karmiel. Les nouveaux immigrants remarquent d'ailleurs avec grand amusement cette trajectoire des Odessites car elle comporte le trait très particulier de s'effectuer pour beaucoup d'entre eux en bateau depuis Odessa, ce qui lui confère un coté très pittoresque. D'autres personnes interrogées avancent plus directement vers une caractérisation d'ordre ethnique de ces comportements. Elles différencient nettement les espaces géorgiens des autres et soulignent notamment que les villes de Lod et Ashdod sont leurs espaces privilégiés. Ce type de vision de l'espace est toutefois peu répandu.

A l'échelle intra-urbaine, les personnes interrogées ont fait remarquer le caractère très diffus de la répartition spatiale au sein de la ville de Jérusalem et ont noté que la résidence des migrants était avant tout liée aux disponibilités du marché immobilier et à des considérations économiques. Par conséquent, les immigrants se localisent, pour Jérusalem, dans le parc immobilier, en majorité locatif, du centre ville et dans celui des espaces de croissance de l'agglomération comme Pisgat Zeev, Neve Yaacov $^{52}$ (où résident de nombreux Géorgiens) et Maaleh Adummim (ville nouvelle de Cisjordanie) où une plus forte proportion de propriétaires est remarquable. Dans une moindre mesure, cette répartition interne à Jérusalem, relève de considérations personnelles que l'on peut toutefois caractériser. Ainsi, il s'avère que les migrants originaires de Kiev en particulier, et principalement ceux qui se définissent comme membres de l'intelligentsia, préfèrent résider dans les quartiers aisés de Jérusalem car

52 Nouveaux quartiers résidentiels au nord-est de Jérusalem dans les limites post-1967 de l'agglomération. 
ils considèrent qu'ils ne peuvent, résider dans des lieux plus modestes. Ils recherchent donc des logements dans des quartiers tels que Rehavia ou Baka. Ce choix de résidence, semble-t-il, leur « jouerait des tours » car ces deux quartiers sont habités pour l'un, par des personnes originaires d'Allemagne depuis longtemps immigrées et quelque peu renfermées sur elles-mêmes, et pour l'autre par des personnes d'origine américaine ou française avec lesquelles les immigrants ont notamment peu de points linguistiques communs. En ce qui concerne Tel Aviv, on notera que les Juifs d'Asie Centrale sont, en majorité, regroupés dans le quartier populaire de Shapira. Les liens familiaux, très forts au sein de cette communauté, et notamment au sein de celle des Juifs du Boukhara, sont à l'origine d'un tel regroupement, et historiquement, ce quartier a toujours constitué un pôle d'accueil priviligié pour ce groupe.

\section{Une fragilisation de l'ethos national ?}

Une question primordiale est celle de l'incidence de cet essor démographique exogène dans la structuration de l'ethos national. La vague migratoire d'ex-URSS, de par son importance, a amené Israël à renouer avec les thèmes qui ont été, par le passé, à l'origine d'intenses tensions inter-communautaires. Très rapidement, au delà de la joie que fut la libération des Juifs d'ex-URSS pour la société israélienne, cette aliya principalement d'origine ashkénaze s'est révélée être la source d'une réactivation du "vieux clivage séfarade/ashkénaze " que la « synthèse sabraïque " avait su estomper. Cette réaction, face à un événement d'importance historique dans le déroulement du continuum, a laissé transparaître la fragilité de cette société. Néanmoins, cette crispation identitaire était sans réelle gravité car elle relevait apparemment plus de tensions d'ordre socio-économique que communautaire. Le nouvel immigrant, chéri par le gouvernement ${ }^{53}$, s'est trouvé dans une situation de favorisé qui était difficilement acceptable pour nombre de jeunes familles de sabarim - bien souvent d'origine séfarade - en situation précaire.

A l'inverse, la capacité des sabarim à dépasser ces clivages d'ordre communautaire a, semble-t-il, contribué à tarir quelque peu le sentiment de communauté de destin si l'on en juge par l'intérêt que portent ceux-ci à l'immigration. Au cours des vingt-cinq dernières années, l'importance accordée à l'immigration n'a cessé de s'éroder. La population israélienne, qui affirmait à plus de $87 \%$ en 1973 que l'immigration était d'une haute importance pour le pays, n'était plus que de $72 \%$ en 1979 et de $66 \%$ en 1994-1995. Cette manifestation d'un désintérêt pour l'immigration est d'autant plus troublante qu'un regain d'attention s'était exprimé lors des premières années de l'aliya d'ex-URSS pour disparaître aussi rapidement comme si cette nouvelle vague migratoire ne correspondait plus aux attentes de la société israélienne. ${ }^{54}$

53 Dans le cadre de l'intégration directe, chaque nouvel immigrant reçoit une aide nommée «panier d'absorption » lui permettant de faire face aux frais et besoins inhérents à l'entrée dans une nouvelle société (logements, équipements, et autres) et d'acquérir une certaine liberté pour chercher un emploi et s'établir dans le pays.

54 Ministry of Immigrant Absorption, Op. Cit., p. 57. 
De fait, le continuum migratoire semble aujourd'hui perdre la force, la puissance identitaire et la fibre mobilisatrice qui le caractérisaient encore il y a à peine cinquante ans. S'agit-il pour autant de la fin de celui-ci ? La rupture de l'harmonie décelée dans notre lecture du continuum semble davantage une ouverture, dont la clef résiderait dans l'émancipation d'Israël où, comme l'a souligné Doris Bensimon, de la trilogie « Dieu, la Torah, le peuple " $\$ 5$, la dimension "peuple » a été plus fortement retenue, et dont le propos est d'incarner la "normalité » du peuple juif. Un cheminement vers une "normalité » qui procéderait d'une rencontre, celle «des. fils et des filles, et plus sûrement encore des petits-fils et des petites-filles des premiers immigrants par le biais des mariages mixtes, du service militaire, de la scolarisation d'Etat, et de la dynamique générale de la vie quotidienne $\gg .{ }^{56}$ Par cette rencontre progressive des enfants d'Israël s'élabore une nouvelle génération d'Israéliens où vont devoir se fondre ou se définir autrement les immigrants. Ce nouveau creuset d'intégration est structuré autour de trois populations qui peuvent être caractérisées schématiquement ainsi :

- la première serait celle des jeunes que l'on voit arpenter les rues de Tel Aviv. Cette population a soif de cette normalité qu'elle puise, peut-être à outrance, dans la " culture américaine ». Le sionisme lui apparaît comme un caractère fossile de la société israélienne, l'armée ne lui semble plus être le lieu privilégié pour exprimer son patriotisme et le judaïsme n'est plus pour elle qu'une voie parmi d'autres dans la quête de soi-même. La multiplication des «voyages initiatiques » en Asie après la période militaire (13000 visas délivrés en 95 pour l'Inde), ou bien encore le développement de centres de méditations asiatiques ou New Age, en sont les expressions actuelles. ${ }^{57}$ Cette quête reflète le rejet par ces sabarim du "caractère moyenâgeux du judaïsme en Israël » et affirme la difficulté pour ces jeunes à supporter la charge spirituelle que lui inflige la diaspora. Un devoir de pureté, de modèle lui serait imposé par la diaspora, dans l'idée que cette dernière ne désire pas que le centre vienne à lui ressembler de peur de ne plus savoir elle-même ce à quoi elle devrait ressembler. Cette population répugne à penser que son pays puisse être « un musée du peuple juif » et cherche avec ardeur toute expression de la modernité. Une jeunesse qui ressemble, par bien des traits, à celles des grandes agglomérations occidentales.

- la seconde que nous pourrions définir par opposition à la première, est celle de Jérusalem, beaucoup plus empreinte de tradition. Elle exprime plus la recherche d'une alternative que d'un rejet, une voie entre cette incessante quête de modernité et la rigueur de l'orthodoxie. Nombre de ces jeunes ont trouvé sur la voie de la normalisation, une alternative dans le renouveau des partis religieux. La nette croissance du parti $S_{h a s}{ }^{58}$ au cours des dernières élections en fut un parfait exemple.

55 Bensimon (D.), Les Juifs dans le monde au tournant du xxr siècle, Albin Michel, Paris (Présences du Judaïsme, $\mathrm{N}^{\circ} 13$ ), 1994, p.106.

56 Vital (D.), « De l’identité Israélienne », Pardès, N 18, Cerf, Paris, p. 69.

57 Pour plus d'informations, la lecture du numéro de Dialogues, $\mathrm{N}^{\circ} 5$ - Hiver 96 , consacré à «Israël : la crise identitaire» sera d'un riche apport. Notamment l'article de Myriam Darmoni, "La jeunesse israélienne a d'autres rêves ".

58 Shas, parti ultra-orthodoxe séfarade ayant obtenu 10 sièges à la Knesset lors des dernières élections israéliennes en 1996. 
Ces jeunes trouvent auprès de tels partis religieux une oreille attentive à qui confier leurs doutes. Ces partis incarnent une stabilité rassurante surtout pour ceux issus du "second Israël ", oriental, pour qui l'intégration à la société israélienne n'est pas toujours chose facile. Cette dynamique, une fois de plus, n'est pas si distante de celle qui mène une jeunesse européenne vers les mouvements dits du « renouveau charismatique ».

- la dernière constitue un ensemble plus hétérogène que l'on peut subdiviser en deux sous-populations. La première serait celle qui réside dans les espaces secondaires d'Israël où la quête de normalisation n'apparaît pas comme une priorité et où, en tout cas elle se réalise sans être pensée comme un but ultime. Cette population en marge de l'effervescence des grands centres urbains, constitue une entité essentielle dans cette route vers la normalisation car elle est productrice du lien social. Elle apporte la cohésion et l'équilibre à la société en absorbant les turbulences nées des "excès» des populations précitées évitant ainsi le schisme. Cette population, de condition sociale souvent modeste, offre le soutien nécessaire aux nouveaux immigrants pourtant perçus aux premières heures de l'aliya comme un "ennemi », un obstacle à leur ascension sociale. En ce milieu se réalise une part importante de l'intégration, trop souvent oblitérée par les problèmes plus médiatiques des élites d'exURSS qui résident à Jérusalem ou Tel Aviv. L'autre population est constituée par les ultra-orthodoxes que l'Occident considère trop facilement comme l'image d'Israël et dont les quartiers de Méa Shéarim pour Jérusalem et Bnei Braq en banlieue de Tel Aviv constituent les centralités. Toutefois, n'oublions pas que si ces espaces se caractérisent par une ultra-orthoxie qui semble d'une autre époque, ils ne sauraient être symboles d'archaïsme. Ces mondes traditionalistes ont également opéré une normalisation qui en a fait des lieux dynamiques et ouverts sur le monde, des espaces où la mobilisation des réseaux communautaires et les échanges se font avec une réelle intensité par des moyens d'une grande modernité.

\section{CONCLUSION - Israël : Un pays d'immigration comme les autres ?}

Répondre à une telle interrogation est malaisée, tentons toutefois d'esquisser l'« évolution » d'Israël à travers deux points de vue : l'un extrinsèque au peuple juif, l'autre véritablement propre à sa personne, et totalement en opposition l'un et l'autre.

En premier lieu, l'affaiblissement du continuum migratoire est renforcé par le fait qu'Israël se trouve pris dans le courant des mobilités générées par le différentiel de développement économique au sein de la planète. Actuellement, le débat sur l'entrée de travailleurs étrangers et de «touristes » issus de Roumanie, d'Afrique (Ghana, Nigeria, Sierra Léone), d'Amérique du Sud (Colombie), d'Asie (Philippines, Inde), et plus récemment d'Egypte, de Jordanie et de Turquie pour ne citer que les flux les plus importants, est de plus en plus intense. Les autorités israéliennes découvrent avec étonnement que ces immigrants légaux (plus de 100000 personnes) et illégaux ont des 
comportements qui laissent à penser qu'ils ne désirent pas repartir. ${ }^{59}$ Les autorités notent que ces résidents font venir leurs familles, qu'ils apprennent rapidement l'hébreu, qu'ils ont des enfants en Israël, et que cette seconde génération demandera bientôt les mêmes droits que les Israéliens. En un mot, l'Etat d'Israël découvre qu'il est devenu un pays attractif pour nombre de pays en développement. Ce constat n'est pas sans créer de troubles dans la société israélienne. Israël se trouve fragilisé par ces nouvelles mobilités et se découvre un rôle inattendu de périphérie dans le sens où, comme le définit M. F. Durand, l'ouverture de telles filières migratoires marque "l'entrée d'une périphérie dans le système centre ${ }^{60}{ }^{60}$ Même si l'Etat d'Israël est loin de perdre son identité, il va devoir composer, peut-être contre son gré, avec celles des nouveaux venus. Néanmoins, Israël semble attiré par les conceptions européennes en matière migratoire, lesquelles, comme le souligne Didier BIGO, font qu' «être différent revient potentiellement à attenter à l'identité nationale, à menacer la sécurité intérieure $(\ldots){ }^{61}{ }^{61}$

En second lieu, outre les signes révélateurs d'une prise de recul de la société israélienne affirmant ainsi l'ère post-sioniste, les vagues migratoires des années quatrevingt dix ont d'une certaine manière reconsolidé les liens entre le centre et la périphérie. Du côté du centre, l'idée d'une rupture d'Israël avec sa diaspora, l'affirmation qu'un ethos national aurait pris l'ascendant sur l'unité de cette grande entité, le peuple juif, ne semblent pas affirmées. Du côté de la périphérie, malgré l'affaiblissement du centre dans sa conception sioniste d'espace d'accueil, et par voie de conséquence la raréfaction des moments d'expression de la diaspora, cette dernière s'est montrée fidèle et prompte à réagir ("L'Opération Exodus», organisée pour soutenir Israël dans l'intégration des immigrants d'ex-URSS, a permis la récolte, entre 1990 et 1994, de 844 millions de dollars). Cet espace relationnel se caractérise par une forme de «supraterritorialité » que sous-tendrait l'allégeance de la périphérie à son centre et dont les rapports avec ce dernier seraient institutionnalisés par un organe international et "supranational», représenté par le couple Organisation Sioniste Mondiale/Agence Juive. Les responsables de ces organisations n'ont d'ailleurs pas manqué de rappeler que «s'il existe une chose qui plus que les autres peut symboliser

59 Cf. article «The new face of Israel ", The Jerusalem Report, August 22, 1996. Les filières roumaines et asiatiques sont une conséquence de la fermenture des frontières israéliennes aux travailleurs palestiniens pour cause de sécurité intérieure. De ce fait l'Etat nécessitait de la main d'oeuvre pour les activités agricoles et de constructions. Les personnes d'origine asiatique sont également employées, comme dans de nombreux pays européens comme l'Espagne, dans des activités d'aide aux personnes âgées. Les entrées africaines sont considérées par certains comme une conséquence directe des Accords d'Oslo qui ont entrainés l'établissement de relations diplomatiques avec les Etats africains. Ces immigrés travaillent en majorité dans les services de nettoyage et résident dans la région de Tel Aviv. Une estimation raisonnable des clandestins se situerait aux environs de 30 à 40000 .

60 In Badie (B.), Withol de Wenden (C.) (ed.), Le défi migratoire ? Questions de relations internationales, Presses de la Fondation Nationale des Sciences Politiques, Paris (Le temps mondial), 1994.

61 Bigo (D.), «Sécurité, immigration et contrôle social. L'archipel des polices », Le Monde Diplomatique, octobre 1996 
la communauté de destin et d'actions du Peuple juif en Israël et en diaspora, c'est bien l'"Opération Exodus" ».

Cette société israélienne diversifiée est donc amenée à travers ses propres logiques à composer avec sa diaspora et par là même à valider ou invalider cette métonymie dont nous parlions en introduction. Cette négociation est d'autant plus difficile à opérer que la diaspora s'est également compartimentée, complexifiée. Le concept de diaspora juive paraît davantage comme une nébuleuse qu'il serait préférable de définir comme des "établissements diasporiques ${ }^{62}$ qui sont autant de territoires aux identités diverses susceptibles d'être mobilisés par les réseaux transnationaux. Les articles publiés dans la presse israélienne sous des titres comme : «Qui parle pour nous ?» ou " Qui sont les acteurs? ? reflètent bien cette difficulté de définition. En effet, quelle est donc cette diaspora : celle des hommes influents de l'AIPAC, celle des Loubavitch, alors qu'en 1990 seulement un tiers des Juifs américains étaient membres d'une synagogue, celle dont la moitié des unions se fait à l'extérieur de la communauté et dont seulement un quart des enfants se considère comme juif ou bien encore cette diaspora «immobile» qui paradoxalement manifeste lorsque les orthodoxes essaient de restreindre le champ d'application de la loi du Retour? Comme le démontre la multiplicité des facettes de cette diaspora, il est bien difficile d'éluder la très actuelle question du futur des rapports entre centre et périphérie. Néanmoins les éléments de réponse se trouvent nécessairement pour le géographe dans l'étude de la territorialité juive, dans le sens où celle-ci révélera chez ces deux protagonistes « l'interaction de leurs représentations et de leurs comportements dans leur relation à l'espace $\gg .{ }^{63}$ En termes conceptuels, le territoire des diasporas, dont l'une des formes archétypales serait celle de la diaspora juive, pourrait être défini comme un espace culturel dont la mémoire collective en constitue les contours, où la non-mobilité fait sens et l'allégeance le signifie.

Cet angle d'étude géographique permet d'identifier nombre de logiques qui régissent les rapports "centre-périphérie » et régulent ainsi le déroulement du continuum migratoire. En l'absence de mobilités ou de mouvements de faible amplitude, et outre les « artefacts migratoires » de la fin 90, l'étude de la territorialité juive apparaît plus que déterminante. Elle est l'instrument nécessaire pour décrire cette nébuleuse qu'est «l'autonomie dans l'appartenance ", "l'émancipation dans la communauté de destin », mise en valeur dans les actes volontaires d'ingérence de la diaspora dans la société israélienne et réciproquement. Cette confusion qui oscille entre l'éternel sentiment d'une absence de centralité et l'impression d'une réelle territorialisation des espaces, démontre l'existence d'une territorialité - voire d'une « supraterritorialité » — tant l'ingérence du centre ou de la périphérie semble échapper à l'Etat-nation qu'elle implique.

Je tiens à remercier Mmes Eléonore Plotkine, Danielle Storper Perez et Florence Heymann pour leur aide dans la réalisation de cet article.

62 Cf. les travaux d'Alain Medam.

63 Piveteau J.L., «La territorialité des Hébreux : l'affaire d'un petit peuple il y a longtemps, ou un cas d'école pour le $\Pi \mathrm{I}^{\mathrm{e}}$ millénaire ? », L'espace géographique, $\mathrm{N}^{\circ} 1,1993$. 


\section{De l'Aliya à l'immigration ou la lecture d'un continuum migratoire}

\section{William BERTHOMIERE}

Ychouv, Aliya, «L'Etat des Juifs », Israël, ces mots résument le propos de notre article qui vise à retracer les trajectoires migratoires qui se sont succédées depuis la création de l'Etat jusqu'à nos jours. De par la diversité et l'importance de ces flux migratoires, nous avons entrepris de lire ces mobilités dans l'idée du déroulement d'un continuum migratoire où la dialectique « centre -périphérie «s'est au fil du temps, complexifiée et diversifiée.

Ainsi, notre lecture présente les temps majeurs de ce continuum à travers les dynamiques des mobilités juives et leurs impacts dans l'édification de l'ethos national en Israël. Un tel prisme d'observation nous conduira inévitablement vers une analyse des mutations dans les rapports «Israël/diaspora » et vers l'identification d'une territorialité juive post-sioniste.

\section{From Aliya to immigration or the reading of a migratory continuum}

\section{William BERTHOMIERE}

Ychouv, Aliya, "The Jewish State », Israel, these words summarize the aim of our article where we try to redraw the migratory trajectories which followed one another from the birth of the State until now. Given the diversity and the importance of these migratory flows, we have decided to read these mobilities with the idea of a course of the migratory continuum in which the dialectic "centre-periphery " has with time become more complicated and more diversified.

Our reading presents the main periods of this continuum through a description of the Jewish mobilities and their impacts on the edification of the Israeli national ethos. Such prism of observation will inevitably lead us to analyze the transformation in the «Israel-diaspora " relations and to identify an post-zionist jewish territoriality.

\section{De l'Aliya a la inmigración, o la lectura de un continuum migratorio}

\section{William BERTHOMIERE}

Ychouv, Aliya, «El Estado de los Jud'os », Israel, estas palabras resumen al propósito de nuestro art'culo que se propone recordar las trayectorias migratorias que se sucedieron desde la creación del Estado hasta hoy. La diversidad y la importancia de los flujos migratorios nos permite leer estas movilidades con la idea del desarrollo de un continuum migratorio en el cual la dialéctica "centro-periferia », se diversifica y se hace más compleja a través el tiempo.

As' pues, nuestra lectura presenta los momentos cruciales del continuum a través de las dinámicas de las movilidades jud'as y de sus impactos en la construcción del ethos nacional en Israel. Este prisma de observación nos conduce inevitablemente al análisis de las mutaciones de la relación «Israel/diáspora » y a la identificación de una territorialidad jud’a post-sionista. 